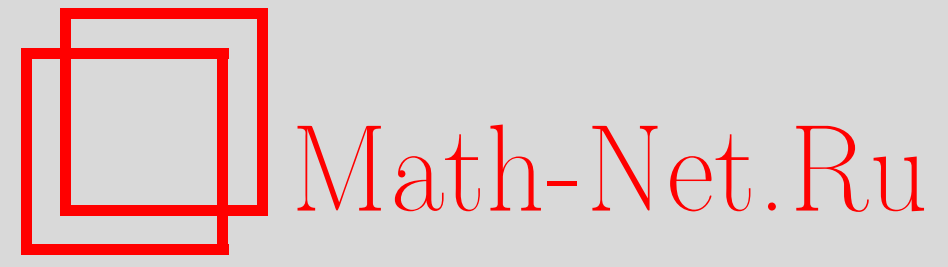

Ч. Су, Т. Ху, Х. Лян, О логарифмическом законе для строго стационарных отрицательно ассоциированных серий, Теория вероятн. и ее примен., 2001, том 46, выпуск 2, 397-407

DOI: https://doi.org/10.4213/tvp3931

Использование Общероссийского математического портала MathNet.Ru подразумевает, что вы прочитали и согласны с пользовательским соглашением

http://www . mathnet.ru/rus/agreement

Параметры загрузки:

IP : 54.80 .97 .219

26 апреля 2023 г., $11: 36: 04$

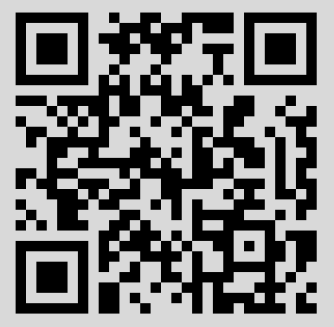


8. Korshunov D. A. On distribution tail of the maximum of a random walk. - Stochastic Process. Appl., 1997, v. 72, № 1, p. 97-103.

9. Sgibnev M.S. On the distribution of the maxima of partial sums. - Statist. Probab. Lett., 1996, v. 28, № 3, p. 235-238.

10. Teugels J. L. The class of subexponential distributions. - Ann. Probab., 1975, v. 3, № 6, p. 1000-1011.

11. Veraverbeke $N$. Asymptotic behavior of Wiener-Hopf factors of a random walk. Stochastic Process. Appl., 1977, v. 5, № 1, p. 27-37.

Поступила в редакцию 19.X.1998

(C) $2001 \mathrm{r}$.

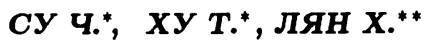

\section{О ЛОГАРИФМИЧЕСКОМ ЗАКОНЕ ДЛЯ СТРОГО СТАЦИОНАРНЫХ ОТРИЦАТЕЛЬНО АССОЦИИРОВАННЫХ СЕРИЙ ${ }^{1)}$}

Доказан логарифмический закон для строго стационарных и отрицательно ассоциированных серий случайных величин, аналогичный логарифмическому закону для независимых одинаково распределенных случайных величин. Использована формула Парсеваля из теории преобразований Фурье для оценки близости между отрицательно ассоциированными и независимыми случайными величинами. Подчеркивается польза применения использованных методов для дохазательства предельных теорем для отрицательно ассоциированных случайных величин.

Ключевые слова и фразы: отрицательно ассоциированная последовательность, серия; логарифмический закон, формула Парсеваля, преобразование Фурье.

0. Введение. Семейство случайных величин $\left\{X_{i}, i=1, \ldots, n\right\}$ называется отричательно ассочиированяым (NA), если для любых двух непересекающихся непустых подмножеств $A$ и $B$ множества $\{1,2, \ldots, n\}$ выполняется неравенство

$$
\operatorname{cov}\left(f_{1}\left(X_{i}, i \in A\right), f_{2}\left(X_{j}, j \in B\right)\right) \leqslant 0,
$$

где $f_{1}$ и $f_{2}$ - покоординатно возрастающие функции такие, что ковариация существует.

Бесконечное множество случайных величин называется отрицательно ассоциированным, если любое конечное его подмножество обладает этим свойством. Свойство множества быть отрицательно ассоциированным также называют отрицательяой ассочиนроваяностью. Это определение было введено Аламом и Сахсеном [1], а затем детально изучалось Йо-Девом и Прошаном [3]. Понятие NA привлекает все большее

Su Chun, Hu Taizhong, Liang Hanying. On the logarithm law for strictly stationary and negatively associated arrays.

(C) Перевод на русский язык, Научное издательство «ТВП», 2001.

* Department of Statistics and Finance, University of Science and Technology of China, Hefei, Anhui 230026, China; e-mail: suchun@ustc.edu.cn, thu@ustc.edu.cn

** Department of Applied Mathematics Tongji University, Shanghai 200092, China.

1) Работа выполнена при поддержке NSF (гранты 19671078 и 19701030), the Doctoral Program Foundation of Institute of High Education и Chinese Academy of Sciences. 
внимание благодаря его широкой применимости в многомерном статистическом анализе и теории надежности. Был установлен ряд предельных закономерностей для последовательностей случайных величин, обладающих свойством NA. Эти свойства аналогичны свойствам, которыми обладают последовательности независимых случайных величин. Например, Ньюмен [6], а также Су и Чи [12] доказали центральную предельную теорему, Матула [5] доказал теорему о трех рядах, Шао [11] и Су с соавторами [13] доказали неравенства для моментов и слабый принцип инвариантности. Некоторые из этих результатов были перенесены на последовательности серий случайных величин, обладающие свойством NA.

Ли с соавторами [4] отметили, что закон повторного логарифма может не выполняться для последовательностей серий независимых одинаково распределенных в каждой серии случайных величин. Ху и Вебер [2], а также Ки [9] изучали логарифмический закон для последовательностей серий независимых в каждой серии случайных величин. Более точно, пусть $\left\{X_{n k}, k=1,2, \ldots, n ; n \in \mathbf{N}\right\}$ - последовательность серий независимых одинаково распределенных в каждой серии случайных величин. В [2] доказано, что

$$
\limsup _{n \rightarrow \infty} \frac{\left|\sum_{k=1}^{n} X_{n k}\right|}{\sqrt{2 n \log n}}=1 \text { п.в., }
$$

если $\mathbf{E} X_{11}=0, \mathbf{E} X_{11}^{2}=1$ и $\mathbf{E} X_{11}^{4}<\infty$. Позже Ки [9] доказал, что (0.1) выполняется тогда и только тогда, когда

$$
\mathbf{E} X_{11}=0, \quad \mathbf{E} X_{11}^{2}=1 \quad \text { и } \quad \mathbf{E}\left[X_{11}^{4}\left(\log \left|X_{11}\right|\right)^{-2}\right]<\infty
$$

Здесь и далее $\log x$ обозначает $\ln (\max (x, e))$ для всех $x>0$.

Цель настоящей статьи - доказать логарифмический закон для строго стационарных последовательностей случайных величин, обладающих свойством NA, при условиях, аналогичных условиям (0.2).

1. Проблема и модель. Пусть $\xi_{0}, \xi_{1}, \xi_{2}, \ldots$ - строго стационарная последовательность случайных величин, обладающая свойством NA. Пусть $\left\{X_{n k}: k=1, \ldots, n\right.$; $n \in \mathbf{N}\}$ - последовательность серий случайных величин со свойством NA, для которой выполняется условие

$$
\left(X_{n 1}, X_{n 2}, \ldots, X_{n n}\right) \stackrel{d}{=}\left(\xi_{1}, \xi_{2}, \ldots, \xi_{n}\right), \quad n \in \mathbf{N}
$$

где $\stackrel{\mathrm{d}}{=}$ обозначает равенство распределений. Положим $S_{n}=\sum_{k=1}^{n} X_{n k}$. Формулировка основного результата статьи включает следующие три предположения:

$1^{\circ} . \mathbf{E}\left[\xi_{0}^{4}\left(\log ^{2}\left|\xi_{0}\right|\right)^{-1}\right]<\infty$ и $\mathbf{E} \xi_{0}=0$;

$2^{\circ} . \beta(n)$ убывает и $\beta(n)=O\left(n^{-\theta}\right)$ для некоторого $\theta>5$, где $\beta(n)=-\mathbf{E}\left(\xi_{0} \xi_{n}\right)=$ $-\mathbf{E}\left(\xi_{j} \xi_{j+n}\right), \forall j \in \mathbf{N}$

$3^{\circ} . \sigma^{2} \triangleq \mathbf{E} \xi_{0}^{2}-2 \sum_{n=1}^{\infty} \beta(n)>0$.

На протяжении всей статьи, ради простоты, $c(c<\infty)$ будет обозначать положительную постоянную, которая может принимать разные значения и зависеть от индексов в зависимости от рассматриваемой ситуации.

Теорема 1. Пусть $\left\{\xi_{0}, \xi_{n}, n \in \mathbf{N}\right\} u\left\{X_{n k}, k=1, \ldots, n ; n \in \mathbf{N}\right\}-$ cлучайные величины, упомянутые выне. Тогда

(i) если выполнены условия $1^{\circ}$ и $3^{\circ}$, mо

$$
\limsup _{n \rightarrow \infty} \frac{\left|S_{n}\right|}{\sqrt{2 \sigma^{2} n \log n}} \leqslant 1 \quad \text { n.6.; }
$$

(ii) если выполнены условия $1^{\circ}-3^{\circ}$, то

$$
\limsup _{n \rightarrow \infty} \frac{\left|S_{n}\right|}{\sqrt{2 \sigma^{2} n \log n}}=1 \text { n.e. }
$$

Более того, если выполяено условие $2^{\circ}$, то условия $1^{\circ}$ и $3^{\circ}$ необходимы для выполнения (1.2). 
3 а м е ч а н и е 1. Предположение $2^{\circ}$ является традиционным в такого рода задачах. Например, Пан [7] использует условие $\beta(n)=O\left(e^{-\lambda n}\right)$ для доказательства оценки Берри-Эссеена.

3 а м е ч а и е 2. Пример строго стационарной последовательности, обладаюшей свойством NA, приведен в [13].

Доказательство необходимости условий тривиально. Так как последовательность серий независимых одинаково распределенных в каждой серии случайных величин обладает свойствами строгой стационарности и NA, то из результатов [4] и [10] следует, что предположения $1^{\circ}$ и $3^{\circ}$ необходимы для (1.2), если выполнено условие $2^{\circ}$. Доказательства (1.1) и (1.2) приведены в пп. 4 и 5 соответственно после того, как в пп. 2 и 3 будут доказаны некоторые леммы и другие вспомогательные результаты.

2. Некоторые леммы. Сначала мы напомним некоторые леммы технического характера, которые будут использоваться в следующих пунктах.

Лемма 1 (см. [13]). Пусть $X_{1}, X_{2}, \ldots, X_{n}$ - последовательность случайньх величия со свойством NA maxas, ито $\mathbf{E} X_{k}=0$ u $\mathbf{E}\left|X_{k}\right|^{p}<\infty, k=1,2, \ldots, n, \partial \Omega s$ некоторого $p \geqslant 2$. Пусть $S_{k}=\sum_{i=1}^{k} X_{i}$. Тогда суиествует постоянная $c_{p}$, зависяиая только от $p$, такая, ито

$$
\mathbf{E}\left(\max _{1 \leqslant k \leqslant n}\left|S_{k}\right|\right)^{p} \leqslant c_{p}\left(n \sup _{1 \leqslant i \leqslant n} \mathbf{E}\left|X_{i}\right|^{p}+\left(n \sup _{1 \leqslant j \leqslant n} \mathbf{E} X_{j}^{2}\right)^{p / 2}\right) .
$$

Лемма 2 (см. [11]). Пусть случайкые величияы $X_{1}, X_{2}, \ldots, X_{n}$ обладакт свойством NA и имеют нулевые средние зяачеяия и конечные вторые моменты. Положим $S_{k}=\sum_{i=1}^{k} X_{i} u B_{n}=\sum_{i=1}^{n} \mathbf{E} X_{i}^{2}$. Тогда для всех $x>0$, a.>0u $0<\beta<1$ выполняется неравенство

$$
\begin{aligned}
\mathbf{P}\left\{\max _{1 \leqslant k \leqslant n}\left|S_{k}\right| \geqslant x\right\} \leqslant & 2 \mathbf{P}\left\{\max _{1 \leqslant k \leqslant n}\left|X_{k}\right|>a\right\} \\
& +\frac{2}{1-\beta} \exp \left\{-\frac{x^{2} \beta}{2\left(a x+B_{n}\right)}\left(1+\frac{2}{3} \ln \left(1+\frac{a x}{B_{n}}\right)\right)\right\} .
\end{aligned}
$$

Лемма 3 (см. [6]). Предположим, что $X_{1}, X_{2}, \ldots, X_{n}$ являются отричательно ассочиนрованньми случайньми величинами. Если $\varphi\left(t_{1}, \ldots, t_{n}\right)$ - их совместная харахтеристическая фуккиия $и \varphi_{j}\left(t_{j}\right)$ - харахтеристические функиии отдельных случайных величนн, то

$$
\left|\varphi\left(t_{1}, \ldots, t_{n}\right)-\prod_{j=1}^{n} \varphi_{j}\left(t_{j}\right)\right| \leqslant-2 \sum_{1 \leqslant j<k \leqslant n}\left|t_{j} t_{k}\right| \operatorname{cov}\left(X_{j}, X_{k}\right) .
$$

Лемма 4 (см. [14]). Пусть $0<\alpha \leqslant 1 u\left\{h_{n}, n \in \mathbf{N}\right\}-$ возрастахияая nоследовательность положительных иисел тахая, что $\lim _{n \rightarrow \infty} h_{n}=\infty$. Обозяачим $C^{\alpha}(\mathbf{R})$ множество функиий $f: \mathbf{R} \rightarrow \mathbf{R}$, удовлетворяющих условию

$$
\|f\|_{\alpha} \stackrel{\wedge}{=} \sup \left\{\frac{|f(x)-f(y)|}{|x-y|^{\alpha}}: x, y \in \mathbf{R}, x \neq y\right\}<\infty .
$$

Тогда для любого $0<\varepsilon<\frac{1}{2}$ кайдется последовательность $\left\{f_{n}, n \in \mathbf{N}\right\}$ бесконечно дифференцируемых функиนй ка $\mathbf{R}$ такая, ито

(a) $\left\{f_{n}^{\prime \prime}, n \in \mathbf{N}\right\} \subset C^{\alpha}(\mathbf{R}) u\left\|f_{n}^{\prime \prime}\right\|_{\alpha} \leqslant K h_{n}^{-2-\alpha}$, где $K-$ постоянная, не зависяuas om $n$;

(b) $0 \leqslant f_{n}(x) \leqslant 1, \forall x \in \mathbf{R}$;

(c) $f_{n}=0$ npu $x \leqslant(1-2 \varepsilon) h_{n} u f_{n}=1$ npu $x \geqslant(1-\varepsilon) h_{n}$.

Лемма 5 (см. [10]). Пусть $\left\{X_{n k}, 1 \leqslant k \leqslant n, n \in \mathbf{N}\right\}$ - последовательность серий независимых в каждой серии случайных величия тахих, что $\mathbf{E} X_{n k}=0 u$ $\sigma_{n k}^{2}=\mathbf{E} X_{n k}^{2}<\infty$. Положим $S_{n}=\sum_{k=1}^{n} X_{n k}, \sigma_{n}^{2}=n^{-1} \sum_{k=1}^{n} \sigma_{n k}^{2} u F_{n k}(x)=$ $\mathbf{P}\left\{X_{n k} \leqslant x\right\}, F_{n}(x)=n^{-1} \sum_{k=1}^{n} F_{n k}(x)$. Предположик, что

1) $0<\liminf _{n \rightarrow \infty} \sigma_{n}^{2} \leqslant \limsup _{n \rightarrow \infty} \sigma_{n}^{2}<\infty$; 
2) $\max _{1 \leqslant k \leqslant n} \sigma_{n k}^{2}=O\left(n(\log n)^{-3 / 2}\right)$;

3) $\lim _{n \rightarrow \infty} \int_{x<-\varepsilon \sqrt{n / \log n}} x^{2} d F_{n}(x)=0, \quad \forall \varepsilon>0$;

4) $\lim _{n \rightarrow \infty} n^{-1} \sum_{k=1}^{n} \mathbf{E}\left|X_{n k}\right|^{2+d}<\infty$ длg rexоторого $d>0$.

Тогда для любых с >0 и $\delta>0$ и всех достаточно больиих $n$

$$
\mathbf{P}\left\{S_{n}>c \sqrt{\sigma_{n}^{2} n \log n}\right\} \geqslant n^{-\delta-c^{2} / 2} .
$$

3. Предварительные результаты. 1 . Пусть $\left\{\xi_{0}, \xi_{n}, n \in \mathbf{N}\right\}-$ строго стационарная последовательность случайных величин, обладающая свойством NA и удовлетворяющая условиям $1^{\circ}$ и $3^{\circ}$ из п. 1 . Положим

$$
\eta_{n}=\frac{1}{\sqrt{n}} \sum_{k=1}^{n} \xi_{k}, \quad n \in \mathbf{N}
$$

Нетрудно видеть, что

$$
\begin{aligned}
\sigma_{n}^{2} & \triangleq \mathbf{E} \eta_{n}^{2}=\mathbf{E} \xi_{0}^{2}+\frac{2}{n} \sum_{1 \leqslant j<l \leqslant n}^{n} \mathbf{E}\left(\xi_{j} \xi_{l}\right)=\mathbf{E} \xi_{0}^{2}-\frac{2}{n} \sum_{l=2}^{n} \sum_{j=1}^{l-1} \beta(l-j) \\
& =\mathbf{E} \xi_{0}^{2}-\frac{2}{n} \sum_{k=1}^{n}(n-k) \beta(k) \geqslant \mathbf{E} \xi_{0}^{2}-2 \sum_{k=1}^{n-1} \beta(k) .
\end{aligned}
$$

(Следует отметить, что $\sigma_{n}^{2}$ в (3.2) отличается от величины, обозначенной той же самой буквой в лемме 5.) Так как $(2 / n) \sum_{k=1}^{n}(n-k) \beta(k) \leqslant 2(n+1)^{-1} \sum_{k=1}^{n+1}(n+1-$ $k) \beta(k)$, то мы имеем

$$
0<\sigma^{2} \leqslant \sigma_{n}^{2} \downarrow \sigma^{2}, \quad n \rightarrow \infty .
$$

Более того, по лемме 1 выполняются соотношения

$$
\gamma_{n} \triangleq \mathbf{E}\left|\eta_{n}\right|^{3}=n^{-3 / 2} \mathbf{E}\left|\sum_{k=1}^{n} \xi_{k}\right|^{3} \leqslant c n^{-3 / 2}\left[n \mathbf{E}\left|\xi_{0}\right|^{3}+\left(n \mathbf{E} \xi_{0}^{2}\right)^{3 / 2}\right] \leqslant c .
$$

На основании сказанного выше мы приходим к следующему результату.

Лемма 6. Предположим, что для каждого $n \in \mathbf{N}$ случайные величияь $Z_{n 1}, Z_{n 2}, \ldots, Z_{n n}$ независимы $u$ их распределекия совпадают с распределением случайной величияы $\eta_{n}$. Положим $T_{n}=\sum_{k=1}^{n} Z_{n k}$. Тогда для любых $c>0$ и $\delta>0$

$$
\mathbf{P}\left\{T_{n} \geqslant c \sqrt{\sigma_{n}^{2} n \log n}\right\} \geqslant n^{-\delta-c^{2} / 2}
$$

для всех достаточно больиих $n$.

Д о х а з а т е л ь с т о о. Утверждение является непосредственным следствием леммы 5, если заметить, что условия 1)-4) выполняются в силу (3.3) и (3.4) в случае независимых одинахово распределенных случайных величин.

2. Пусть $\left\{\xi_{0}, \xi_{n}, n \in \mathbf{N}\right\}$ - последовательность одинаково распределенных случайных величин, обладающая свойством NA (необязательно строго стационарная) и удовлетворяющая условию $1^{\circ}$. Пусть $\left\{m_{n}, n \in \mathbf{N}\right\}$ - последовательность натуральных чисел, удовлетворяющих условию $m_{n}=O\left(n^{1-\delta}\right)$ для некоторого $0<\delta<1$. Положим $\tau^{2}=\mathbf{E} \xi_{0}^{2}$ и $S_{m_{n}}=\sum_{k=1}^{m_{n}} \xi_{k}$.

Лемма 7. Если выполнены перечисленяые выие условия, то для любого $0<$ $\eta<\frac{1}{4}$ мы имеем

$$
\sum_{n=1}^{\infty} \mathbf{P}\left\{\left|S_{m_{n}}\right|>\eta \sqrt{n \log n}\right\}<\infty
$$


Д о к а 3 а т ел в с т в о. Применяя лемму 2 с $x=\eta \sqrt{n \log n}, a=\eta^{2} \sqrt{n / \log n}$ и $\beta=1-\eta$, мы получим

$$
\begin{aligned}
\mathbf{P}\left\{\left|S_{m_{n}}\right|>\eta \sqrt{n \log n}\right\} \leqslant & 2 m_{n} \mathbf{P}\left\{\left|\xi_{0}\right|>\eta^{2} \sqrt{\frac{n}{\log n}}\right\} \\
& +\frac{2}{\eta} \exp \left\{-\frac{\eta^{2}(1-\eta) n \log n}{2\left(\eta^{3} n+\tau^{2} m_{n}\right)}\right\} .
\end{aligned}
$$

Так как $0<\eta<\frac{1}{4}$ и $m_{n} / n \leqslant c n^{-\delta} \longrightarrow 0$, то отсюда следует, что $\alpha \triangleq(1-\eta) /(3 \eta)>1$ и $2\left(\eta^{3} n+\tau^{2} m_{n}\right)<3 \eta^{3} n$ для всех достаточно больших $n$. Таким образом,

$$
\exp \left\{-\frac{\eta^{2}(1-\eta) n \log n}{2\left(\eta^{3} n+\tau^{2} m_{n}\right)}\right\}<n^{-\alpha}
$$

С другой стороны,

$$
\begin{aligned}
\sum_{n=1}^{\infty} m_{n} \mathbf{P}\left\{\left|\xi_{0}\right|>\eta^{2} \sqrt{\frac{n}{\log n}}\right\} & \leqslant c \sum_{n=1}^{\infty} n^{1-\delta} \mathbf{P}\left\{\xi_{0}^{2} \log \left|\xi_{0}\right|>\frac{1}{3} \eta^{4} n\right\} \\
& \leqslant c \mathbf{E}\left|\xi_{0}^{2} \log \right| \xi_{0}||^{2-\delta} \leqslant c \mathbf{E}\left|\xi_{0}\right|^{4-\delta}<\infty .
\end{aligned}
$$

Ясно, что (3.6) следует из (3.7)-(3.9) и предположения $1^{\circ}$.

3. Пусть $\left\{\xi_{0}, \xi_{n}, n \in \mathbf{N}\right\}$ - строго стационарная последовательность случайных величин со свойством NA. Предположим, что она удовлетворяет условиям $1^{\circ}-3^{\circ}$. Обозначим $[x]$ целую часть числа $x$, и пусть $\left\{d_{n}, n \in \mathbb{N}\right\}$ - последовательность натуральных чисел, удовлетворяющих условию $d_{n}=\left[n^{1-\delta}\right]$ для некоторого $0<\delta<1$ такого, что $\rho=(\theta-2)(1-\delta)>3$, где $\theta$ - число, о котором говорится в условии $2^{\circ}$. Для любого $n \in \mathbf{N}$ положим

$$
\begin{gathered}
a_{0}=b_{0}=0 ; \quad a_{1}=n, \quad b_{1}=a_{1}+d_{n} ; \ldots ; \\
a_{j+1}=b_{j}+n, \quad b_{j+1}=a_{j+1}+d_{n}, j \in \mathbf{N}, \\
\eta_{n j}=\frac{1}{\sqrt{n}} \sum_{k=b_{j-1}+1}^{a_{j}} \xi_{k}=\frac{1}{\sqrt{n}} \sum_{k=b_{j-1}+1}^{b_{j-1}+n} \xi_{k}, j=1,2, \ldots, n, \\
\Delta_{n}=-\sum_{1 \leqslant i<j \leqslant n} \mathbf{E}\left(\eta_{n} \mid \eta_{n j}\right) .
\end{gathered}
$$

Тогда для каждого $n$ случайные величины $\eta_{n 1}, \eta_{n 2}, \ldots, \eta_{n n}$ обладают свойством NA и одинаково распределены.

Лемма 8. При условиях, сформулированкых выие,

$$
\sum_{n=1}^{\infty} \sqrt{n \Delta_{n}}<\infty
$$

Дока за те льст то. Мы имеем

$$
\begin{aligned}
\Delta_{n} & =-\sum_{l=1}^{n-1} \mathbf{E}\left[\eta_{n l}\left(\sum_{j=l+1}^{n} \eta_{n j}\right)\right] \leqslant \frac{1}{n} \sum_{l=1}^{n-1}\left(\sum_{k=1}^{n} k \beta\left(d_{n}+k\right)+n \sum_{k \geqslant n+2 d_{n}} \beta(k)\right) \\
& \leqslant \sum_{k=1}^{n} k \beta\left(d_{n}+k\right)+n \sum_{k=n}^{\infty} \beta(k) \leqslant \sum_{k=1}^{d_{n}} k \beta\left(d_{n}\right)+\sum_{k=d_{n}+1}^{n} k \beta(k)+n \sum_{k=n}^{\infty} \beta(k) \\
& \leqslant c\left(d_{n}^{-\theta} \sum_{k=1}^{d_{n}} k+\sum_{k=d_{n}}^{n} k^{1-\theta}+n \sum_{k=n}^{\infty} k^{-\theta}\right) \leqslant c d_{n}^{2-\theta} \leqslant c n^{(2-\theta)(1-\delta)}=c n^{-\rho} .
\end{aligned}
$$

Это влечет (3.13), так как $\rho>3$. 
4. Доказательство первой части теоремы 1. На протяжении этого пункта мы считаем, что выполнены предположения $1^{\circ}$ и $3^{\circ}$. Приступим к доказательству соотношения (1.1) из теоремы 1 . Чтобы доказать (1.1), достаточно показать, что для любого фиксированного $0<\varepsilon<\frac{1}{30} \wedge(4 \sqrt{2} \sigma)^{-1}$ выполняется неравенство

$$
\limsup _{n \rightarrow \infty} \frac{\left|S_{n}\right|}{\sqrt{2 \sigma^{2} n \log n}} \leqslant 1+15 \varepsilon
$$

где $a \wedge b$ обозначает $\min (a, b)$. Чтобы доказать (4.1), в силу леммы Бореля-Кантелли достаточно показать, что

$$
\sum_{n=1}^{\infty} \mathbf{P}\left\{\left|S_{n}\right|>(1+15 \varepsilon) \sqrt{2 \sigma^{2} n \log n}\right\}<\infty .
$$

Из (3.2) и (3.3) вытекает существование $m \in \mathbf{N}$ такого, что

$$
\sigma^{2} \leqslant \sigma_{m}^{2}=\frac{1}{m} \mathbf{E}\left(\sum_{k=1}^{m} \xi_{k}\right)^{2}<\sigma^{2}(1+\varepsilon) .
$$

Положим $k_{n}=m[n / m]$ и $m_{n}=n-k_{n}$. Выполняются соотношения $0 \leqslant m_{n}<$ $m=o\left(n^{1-\delta}\right)$ для любого $0<\delta<1$. В соответствии с леммой 7 , в которой следует положить $\eta=\sqrt{2} \sigma \varepsilon<\frac{1}{4}$, для того чтобы доказать (4.2), достаточно показать, что

$$
\sum_{n=m}^{\infty} \mathbf{P}\left\{\left|S_{k_{n}}\right|>(1+14 \varepsilon) \sqrt{2 \sigma^{2} n \log n}\right\}<\infty .
$$

Положим

$$
\lambda_{n}=\frac{\varepsilon \sigma}{m} \sqrt{\frac{n}{\log n}} \quad \text { и } \quad \rho_{n}=\varepsilon \sqrt{2 \sigma^{2} n \log n} \quad \text { для } n \geqslant m,
$$

а затем определим следующие величины:

$$
\begin{aligned}
X_{n k}^{(1)} & =\left(\left(-\lambda_{n}\right) \vee X_{n k}\right) \wedge \lambda_{n}, \\
X_{n k}^{(2)} & =\left(X_{n k}-\lambda_{n}\right) I_{\left(\lambda_{n}<X_{n k} \leqslant \rho_{n}\right)}, \\
X_{n k}^{(3)} & =\left(X_{n k}+\lambda_{n}\right) I_{\left(-\lambda_{n}>X_{n k} \geqslant-\rho_{n}\right)}, \\
X_{n k}^{(4)} & =\left(X_{n k}+\lambda_{n}\right) I_{\left(X_{n k}<-\rho_{n}\right)}+\left(X_{n k}-\lambda_{n}\right) I_{\left(X_{n k}>\rho_{n}\right)}
\end{aligned}
$$

для $k=1,2, \ldots, k_{n}$ и

$$
S_{k_{n}}^{(j)}=\sum_{k=1}^{k_{n}} X_{n k}^{(j)}, \quad j=1,2,3,4,
$$

где $a \vee b$ обозначает $\max (a, b)$. Таким образом, мы имеем

$$
S_{k_{n}}=\sum_{j=1}^{4} S_{k_{n}}^{(j)} \text {. }
$$

Во-первых,

$$
\begin{aligned}
\sum_{n=m}^{\infty} \mathbf{P}\left\{S_{k_{n}}^{(\mathbf{4})} \neq 0\right\} & \leqslant \sum_{n=m}^{\infty} \mathbf{P}\left\{\bigcup_{k=1}^{k_{n}}\left(\left|X_{n k}\right|>\rho_{n}\right)\right\} \leqslant \sum_{n=1}^{\infty} n \mathbf{P}\left\{\left|\xi_{0}\right|>\rho_{n}\right\} \\
& =\sum_{n=1}^{\infty} n \mathbf{P}\left\{\xi_{0}^{2}>2 \varepsilon^{2} \sigma^{2} n \log n\right\} \triangleq \sum_{n=1}^{\infty} n \mathbf{P}\left\{\xi_{0}^{2}>c n \log n\right\}<\infty .
\end{aligned}
$$

Последнее неравенство вытекает из хорошо известного факта, согласно которому условие $\mathbf{E}\left\{\xi_{0}^{4} / \log ^{2}\left|\xi_{0}\right|\right\}<\infty$ влечет $\sum_{n=m}^{\infty} n \mathbf{P}\left\{\xi_{0}^{2}>c n \log n\right\}<\infty$. 
Во-вторых, из определения величины $X_{n k}^{(2)}$ следует, что $S_{k_{n}}^{(2)}>0$ и

$\mathbf{P}\left\{S_{k_{n}}^{(2)}>3 \varepsilon \sqrt{2 \sigma^{2} n \log n}\right\}=\mathbf{P}\left\{\sum_{k=1}^{k_{n}} X_{n k}^{(2)}>3 \rho_{n}\right\}$

$\leqslant \mathbf{P}\left\{\right.$ найдутся по крайкей мере три $k$ такие, что $\left.X_{n k}^{(2)} \neq 0\right\}$

$\leqslant \sum_{1 \leqslant i<j<k \leqslant k_{n}} \mathbf{P}\left\{X_{n i}>\lambda_{n}, X_{n j}>\lambda_{n}, X_{n k}>\lambda_{n}\right\}$

$\leqslant k_{n}^{3}\left(\mathbf{P}\left\{\xi_{0}>\lambda_{n}\right\}\right)^{3} \quad$ (по свойству NA)

$\leqslant n^{3}\left(\mathbf{P}\left\{\xi_{0}>\frac{\varepsilon \sigma}{m} \sqrt{\frac{n}{\log n}}\right\}\right)^{3} \leqslant c n^{3}\left(\left(\sqrt{\frac{n}{\log n}}\right)^{-3} \mathbf{E}\left|\xi_{0}\right|^{3}\right)^{3}=c n^{-3 / 2} \log ^{9 / 2} n$.

Отсюда следует, что

$$
\sum_{n=m}^{\infty} \mathbf{P}\left\{S_{k_{n}}^{(2)}>3 \varepsilon \sqrt{2 \sigma^{2} n \log n}\right\}<\infty .
$$

Аналогично мы получим

$$
S_{k_{n}}^{(3)} \leqslant 0 \quad \text { и } \quad \sum_{n=m}^{\infty} \mathbf{P}\left\{S_{k_{n}}^{(3)}<-3 \varepsilon \sqrt{2 \sigma^{2} n \log n}\right\}<\infty .
$$

В-третьих, в силу предположения $1^{\circ}$ мы получим

$$
\left|\mathbf{E} S_{k_{n}}^{(1)}\right| \leqslant k_{n}\left|\mathbf{E} X_{n 1}^{(1)}\right| \leqslant n\left\{\lambda_{n} \mathbf{P}\left\{\left|\xi_{0}\right|>\lambda_{n}\right\}+\mathbf{E}\left[\left|\xi_{0}\right| I_{\left(\left|\xi_{0}\right|>\lambda_{n}\right)}\right]\right\}=o(\sqrt{n \log n}) .
$$

Следовательно, утверждение (4.4) будет выполняться, если мы покажем, что

$$
\sum_{n=1}^{\infty} \mathbf{P}\left\{\left|S_{k_{n}}^{(1)}-\mathbf{E} S_{k_{n}}^{(1)}\right|>(1+7 \varepsilon) \sqrt{2 \sigma^{2} n \log n}\right\}<\infty .
$$

Докажем (4.11). Ради простоты положим $l_{n}=[n / m]$. Тогда $k_{n}=m l_{n}$. Определим случайные величины $Y_{n k}=X_{n k}^{(1)}-\mathbf{E} X_{n k}^{(1)}$,

$$
\begin{gathered}
Z_{n j}=\sum_{k=(j-1) m+1}^{j m} Y_{n k}, j=1, \ldots, l_{n}, \\
T_{n}=\sum_{j=1}^{l_{n}} Z_{n j}=\sum_{k=1}^{k_{n}} Y_{n k}=S_{k_{n}}^{(1)}-\mathbf{E} S_{k_{n}}^{(1)} .
\end{gathered}
$$

Очевидно, что случайные величины $\left\{Z_{n 1}, Z_{n 2}, \ldots, Z_{n, l_{n}}\right\}$ обладают свойством NA; для каждого $n$ одинаково распределены и при $n \rightarrow \infty$

$$
\frac{1}{m} \mathbf{E} Z_{n 1}^{2} \rightarrow \sigma_{m}^{2}, \quad \frac{1}{n} \sum_{j=1}^{l_{n}} \mathbf{E} Z_{n j}^{2}=\frac{l_{n}}{n} \mathbf{E} Z_{n 1}^{2}=\frac{k_{n}}{n}\left(\frac{1}{m} \mathbf{E} Z_{n 1}^{2}\right) \rightarrow \sigma_{m}^{2},
$$

что вместе с (4.3) влечет

$$
\sum_{j=1}^{l_{n}} \mathbf{E} Z_{n j}^{2}=l_{n} \mathbf{E} Z_{n 1}^{2}<n \sigma^{2}(1+2 \varepsilon)
$$

для всех достаточно больших $n$. Легко видеть, что из определения случайных величин следует $\left|Z_{n j}\right| \leqslant m\left(\max _{(j-1) m<k \leqslant m j}\left|Y_{n_{k}}\right|\right) \leqslant 2 m \lambda_{n}=2 \varepsilon \sigma \sqrt{n / \log n}, j=1, \ldots, l_{n}$. Следовательно, применяя лемму 2 с $x=(1+7 \varepsilon) \sqrt{2 \sigma^{2} n \log n}, a=2 \varepsilon \sigma \sqrt{n / \log n}$ и 
$\beta=1-\varepsilon$, мы получим

$$
\begin{aligned}
& \mathbf{P}\left\{\left|S_{k_{n}}^{(1)}-\mathbf{E} S_{k_{n}}^{(1)}\right|>(1+7 \varepsilon) \sqrt{2 \sigma^{2} n \log n}\right\}=\mathbf{P}\left\{\left|T_{n}\right|>(1+7 \varepsilon) \sqrt{2 \sigma^{2} n \log n}\right\} \\
& \quad \leqslant \frac{2}{\varepsilon} \exp \left\{-\frac{(1-\varepsilon)(1+7 \varepsilon)^{2} \sigma^{2} n \log n}{(1+7 \varepsilon)\left(2 \sigma^{2} n \log n\right)^{1 / 2}\left(2 m \lambda_{n}\right)+l_{n} \mathbf{E} Z_{n 1}^{2}}\right\} \\
& \quad \leqslant \frac{2}{\varepsilon} \exp \left\{-\frac{(1-\varepsilon)(1+7 \varepsilon)^{2} \log n}{4(1+7 \varepsilon) \varepsilon+1+2 \varepsilon}\right\} \leqslant \frac{2}{\varepsilon} n^{-(1-\varepsilon)(1+7 \varepsilon)^{2}} \leqslant \frac{2}{\varepsilon} n^{-(1+\varepsilon)},
\end{aligned}
$$

что влечет (4.11).

Тем самым, мы завершили доказательство первой части теоремы 1.

5. Доказательство второй части теоремы 1. Теперь мы докажем (1.2) при условиях $1^{\circ}-3^{\circ}$. В силу (1.1) нам нужно доказать только то, что

$$
\limsup _{n \rightarrow \infty} \frac{S_{n}}{\sqrt{2 \sigma^{2} n \log n}} \geqslant 1 \text { п.в., }
$$

что справедливо, если

$$
\limsup _{n \rightarrow \infty} \frac{S_{n}}{\sqrt{2 \sigma^{2} n \log n}} \geqslant 1-5 \varepsilon \quad \text { п.в. }
$$

для любого фиксированного $0<\varepsilon<\frac{1}{30} \wedge(4 \sqrt{2} \sigma)^{-1}$. Таким образом, в силу обобщенной леммы Бореля-Кантелли (см. [8]) достаточно показать, что

$$
\sum_{n=1}^{\infty} \mathbf{P}\left\{S_{n}>(1-5 \varepsilon) \sqrt{2 \sigma^{2} n \log n}\right\}=\infty .
$$

Следующие шесть шагов составляют доказательство (5.3).

Ш а г 1. Предполагая выполненным условие $2^{\circ}$, для $\theta>5$ выберем $\delta \in(0,1)$ такое, что

$$
\rho=(\theta-2)(1-\delta)>3 \text {. }
$$

Положим

$$
d_{n}=\left[n^{1-\delta}\right] \quad \text { и } \quad \tau_{n}=n^{2}+(n-1) d_{n}, \quad \forall n \in \mathbf{N},
$$

и затем определим множество

$$
\begin{aligned}
J_{n} & =\left\{k \in \mathbf{N}: b_{j-1}<k \leqslant a_{j}, j=1,2, \ldots, n\right\} \\
& =\left\{k \in \mathbf{N}: b_{j}<k \leqslant b_{j}+n, j=0,1, \ldots, n-1\right\},
\end{aligned}
$$

где $a_{j}=a_{j}(n)$ и $b_{j}=b_{j}(n)$ определены в (3.10). Кроме того, для каждого натурального числа $l, \tau_{n} \leqslant l<\tau_{n+1}$, мы определим

$$
\begin{gathered}
L=\{1,2, \ldots, l\}-J_{n}, \\
S_{l}^{(1)}=\sum_{k \in J_{n}} X_{l k} \quad \text { и } \quad S_{l}^{(2)}=\sum_{k \in L} X_{l k} .
\end{gathered}
$$

Пусть $\sharp(A)$ обозначает мощность множества $A$. Тогда $\sharp\left(J_{n}\right)=n^{2}, n \in \mathrm{N}$, и

$$
m_{l} \triangleq \sharp(L)<\tau_{n+1}-n^{2}=(n+1)^{2}-n^{2}+n d_{n+1} \leqslant 2 n^{2-\delta}=O\left(l^{1-\delta / 2}\right) .
$$

Так как $S_{l}^{(2)}$ является суммой $m_{l}=O\left(l^{1-\delta / 2}\right)$ одинаково распределенных случайных величин, обладающих свойством NA, то из леммы 7 с $\eta=\sqrt{2} \sigma \varepsilon<\frac{1}{4}$ следует, что

$$
\sum_{l=1}^{\infty} \mathbf{P}\left\{\left|S_{l}^{(2)}\right|>\varepsilon \sqrt{2 \sigma^{2} l \log l}\right\}<\infty .
$$

Заметим, что $S_{l}=S_{l}^{(1)}+S_{l}^{(2)}$ в силу (5.7). Чтобы доказать (5.3), достаточно показать, что

$$
\sum_{l=1}^{\infty} \mathbf{P}\left\{S_{l}^{(1)}>(1-4 \varepsilon) \sqrt{2 \sigma^{2} l \log l}\right\}=\infty .
$$


Ш а г 2. Пусть $\tau_{n}-$ величина, определенная в (5.5). Мы имеем $1<$ $\tau_{n} / n^{2} \leqslant l / n^{2}<\tau_{n+1} / n^{2} \longrightarrow 1, n \rightarrow \infty$, и, следовательно, $\max _{\tau_{n} \leqslant l<\tau_{n+1}} l \log l<$ $(1+\eta)^{2} n^{2} \log n^{2}$ для любого $\eta>0$, когда $n$ велико. Выберем $\eta$ настолько малым, чтобы $(1+\eta)(1-4 \varepsilon)<1-3 \varepsilon$. Таким образом, чтобы доказать $(5.9)$, достаточно показать, что

$$
\sum_{n=1}^{\infty} \sum_{\tau_{n} \leqslant l<\tau_{n+1}} \mathbf{P}\left\{S_{l}^{(1)}>(1-3 \varepsilon) \sqrt{2 \sigma^{2} n^{2} \log n^{2}}\right\}=\infty .
$$

Ш а г 3. Положим

$$
Y_{n, j+1}=\frac{1}{\sqrt{n}} \sum_{k=b_{j+1}}^{b_{j}+n} X_{\tau_{n}, k}, \quad j=0,1, \ldots, n-1 .
$$

Для каждого $l, \tau_{n} \leqslant l<\tau_{n+1}$, мы имеем

$$
\begin{gathered}
\left(\frac{1}{\sqrt{n}} \sum_{k=1}^{n} X_{l k}, \frac{1}{\sqrt{n}} \sum_{k=b_{1}+1}^{b_{1}+n} X_{l k}, \ldots, \frac{1}{\sqrt{n}} \sum_{k=b_{n-1}+1}^{b_{n-1}+n} X_{l k}\right) \\
\stackrel{\mathrm{d}}{=}\left(Y_{n 1}, Y_{n 2}, \ldots, Y_{n n}\right) \stackrel{\mathrm{d}}{=}\left(\eta_{n 1}, \eta_{n 2}, \ldots, \eta_{n n}\right),
\end{gathered}
$$

где $\eta_{n 1}, \ldots, \eta_{n n}$ определены в (3.11). Полагая $V_{n}=\sum_{j=1}^{n} Y_{n j}$, мы получим $S_{l}^{(1)} \stackrel{\mathrm{d}}{=}$ $\sqrt{n} V_{n}, \tau_{n} \leqslant l<\tau_{n+1}$. Заметим, что $\tau_{n+1}-\tau_{n}=O(n)$ в силу (5.5). Чтобы доказать $(5.10)$, достаточно показать, что

$$
\sum_{n=1}^{\infty} n \mathbf{P}\left\{V_{n}>(1-3 \varepsilon) \sqrt{2 \sigma^{2} n \log n^{2}}\right\}=\infty .
$$

Так как $0<\sigma^{2} \leqslant \sigma_{n}^{2}$ для всех $n \geqslant 1$, то достаточно показать, что

$$
\sum_{n=1}^{\infty} n \mathbf{P}\left\{V_{n}>(1-2 \varepsilon) \sqrt{4 \sigma_{n}^{2} n \log n}\right\}=\infty .
$$

Ш а г 4. Для каждого $n$ случайные величины $Z_{n 1}, Z_{n 2}, \ldots, Z_{n n}$ независимы, одинаково распределены и $Z_{n 1} \stackrel{\mathrm{d}}{=} Y_{n 1} \stackrel{\mathrm{d}}{=} \eta_{n 1}$. Положим $T_{n}=\sum_{j=1}^{n} Z_{n j}$. Применяя лемму 6 с $c=2(1-\varepsilon)$ и $\delta=\varepsilon / 2$ в (3.5), мы получим

$$
\sum_{n=1}^{\infty} n \mathbf{P}\left\{T_{n}>(1-\varepsilon) \sqrt{4 \sigma_{n}^{2} n \log n}\right\} \geqslant \sum_{n=1}^{\infty} n^{1-2(1-\varepsilon)^{2}-\epsilon / 2} \geqslant \sum_{n=1}^{\infty} n^{-(1-\varepsilon)}=\infty .
$$

Ш а г 5. Обозначим $h_{n}=\sqrt{4 \sigma_{n}^{2} \log n}$. Из (3.2) и предположения $2^{\circ}$ следует, что $h_{n}$ не убывает и $0<h_{n} / \sqrt{\log n} \longrightarrow 2 \sigma$ при $n \rightarrow \infty$. На основании леммы 4 с $\alpha=1$ мы выберем последовательность $\left\{f_{n}, n \in \mathbf{N}\right\}$ функций на $\mathbf{R}$ со свойствами (a), (b) и (c). Тогда

$$
\begin{aligned}
\mathbf{P}\left\{V_{n}>(1-2 \varepsilon) \sqrt{4 \sigma_{n}^{2} n \log n}\right\} & \geqslant \mathbf{E} f_{n}\left(\frac{V_{n}}{\sqrt{n}}\right) \\
& \geqslant \mathbf{E} f_{n}\left(\frac{T_{n}}{\sqrt{n}}\right)-\left|\mathbf{E} f_{n}\left(\frac{V_{n}}{\sqrt{n}}\right)-\mathbf{E} f_{n}\left(\frac{T_{n}}{\sqrt{n}}\right)\right| \\
& \geqslant \mathbf{P}\left\{T_{n}>(1-\varepsilon) \sqrt{4 \sigma_{n}^{2} n \log n}\right\}-I(n),
\end{aligned}
$$

где

$$
I(n)=\left|\mathbf{E} f_{n}\left(\frac{V_{n}}{\sqrt{n}}\right)-\mathbf{E} f_{n}\left(\frac{T_{n}}{\sqrt{n}}\right)\right| .
$$

Таким образом, утверждение (5.14) будет следовать из (5.15), если мы покажем, что

$$
\sum_{n=1}^{\infty} n I(n)<\infty
$$


Ш а г 6. Обозначим $F_{n}(x)$ и $G_{n}(x)\left(p_{n}(t)\right.$ и $\left.q_{n}(t)\right)$ функции распределения (характеристические функции) случайных величин $V_{n} / \sqrt{n}$ и $T_{n} / \sqrt{n}$ соответственно. Тогда

$$
I(n)=\left|\int_{-\infty}^{\infty} f_{n}(x) d\left(F_{n}(x)-G_{n}(x)\right)\right|=\left|\int_{-\infty}^{\infty} f_{n}^{\prime}(x)\left(F_{n}(x)-G_{n}(x)\right) d x\right| .
$$

Так как $f_{n}^{\prime}(x)=f_{n}^{\prime \prime}(x)=0$ на $\left(-\infty,(1-2 \varepsilon) h_{n}\right] \cup\left[(1-\varepsilon) h_{n}, \infty\right)$ и $\left\|f_{n}^{\prime \prime}\right\|_{1} \leqslant c h_{n}^{-3}$, то по лемме 4 (a) мы имеем

$$
\left|f_{n}^{\prime \prime}(x)\right|=\left|f_{n}^{\prime \prime}(x)-f_{n}^{\prime \prime}\left((1-2 \varepsilon) h_{n}\right)\right| \leqslant c h_{n}^{-3}\left|x-(1-2 \varepsilon) h_{n}\right|
$$

на $\left((1-2 \varepsilon) h_{n},(1-\varepsilon) h_{n}\right)$. Ясно, что $f_{n}^{\prime} \in L_{2}(\mathbf{R})$, где $L_{2}(\mathbf{R})-$ множество квадратично интегрируемых функций, определенных на $\mathbf{R}$. Если положить

To

$$
\psi_{n}(t)=\int_{-\infty}^{\infty} e^{\mathrm{i} t x} f_{n}^{\prime}(x) d x=-\frac{1}{\mathrm{i} t} \int_{-\infty}^{\infty} e^{\mathrm{i} t x} f_{n}^{\prime \prime}(x) d x
$$

$$
\left|\psi_{n}(t)\right|=\frac{1}{|t|} \int_{(1-2 \varepsilon) h_{n}}^{(1-\varepsilon) h_{n}}\left|f_{n}^{\prime \prime}(x)\right| d x \leqslant \frac{c}{|t|} h_{n}^{-3} \int_{0}^{h_{n} \varepsilon} x d x=\frac{c}{h_{n}|t|} .
$$

Следовательно, $\psi_{n} \in L_{2}(\mathbf{R})$.

На основании свойств случайных величин, обладающих свойством NA, мы заключаем, что

и для $x>1$

$$
\begin{aligned}
& \mathbf{E}\left(\frac{V_{n}}{\sqrt{n}}\right)^{2}=\frac{1}{n} \mathbf{E}\left(\sum_{j=1}^{n} \eta_{n j}\right)^{2} \leqslant \frac{1}{n} \sum_{j=1}^{n} \mathbf{E} \eta_{n j}^{2}=\mathbf{E} \eta_{n 1}^{2}=\frac{1}{n} \mathbf{E}\left(\sum_{k=1}^{n} \xi_{k}\right)^{2} \leqslant \mathbf{E} \xi_{0}^{2} \\
& \mathbf{E}\left(\frac{T_{n}}{\sqrt{n}}\right)^{2}=\mathbf{E} \eta_{n 1}^{2} \leqslant \mathbf{E} \xi_{0}^{2}
\end{aligned}
$$

$$
\begin{aligned}
\left|F_{n}(x)-G_{n}(x)\right| & \leqslant \mathbf{P}\left\{\left|\frac{T_{n}}{\sqrt{n}}\right| \geqslant x\right\}+\mathbf{P}\left\{\left|\frac{V_{n}}{\sqrt{n}}\right| \geqslant x\right\} \\
& \leqslant \frac{1}{x^{2}}\left[\mathbf{E}\left(\frac{T_{n}}{\sqrt{n}}\right)^{2}+\mathbf{E}\left(\frac{V_{n}}{\sqrt{n}}\right)^{2}\right] \leqslant \frac{c}{x^{2}}
\end{aligned}
$$

что влечет принадлежность функций $B_{n}(x) \triangleq F_{n}(x)-G_{n}(x)$ и $\varphi_{n}(t) \triangleq-(\mathrm{i} t)^{-1}\left(p_{n}(t)-\right.$ $\left.q_{n}(t)\right)$ множеству $L_{2}(\mathbf{R})$. Применяя формулу Парсеваля (см. [8]) к (5.18) и принимая во внимание (5.19), мы получим

$$
I(n)=\frac{1}{2 \pi}\left|\int_{-\infty}^{\infty}\left(p_{n}(t)-q_{n}(t)\right) \frac{\bar{\psi}_{n}(t)}{t} d t\right| \leqslant \frac{c}{h_{n}} \int_{-\infty}^{\infty}\left|p_{n}(t)-q_{n}(t)\right| \frac{1}{t^{2}} d t
$$

Tak кak

и

$$
p_{n}(t)=\mathbf{E}\left[\exp \left\{\frac{\mathrm{i} t}{\sqrt{n}} \sum_{j=1}^{n} \eta_{n j}\right\}\right], \quad q_{n}(t)=\prod_{j=1}^{n} \mathbf{E}\left[\exp \left\{\frac{\mathrm{i} t \eta_{n j}}{\sqrt{n}}\right\}\right]
$$

$$
\left|p_{n}(t)-q_{n}(t)\right| \leqslant 2, \quad \forall t \in \mathbf{R},
$$

мы заключаем, в силу леммы 3 и (3.12), что

$$
\left|p_{n}(t)-q_{n}(t)\right| \leqslant \frac{2 t^{2}}{n} \Delta_{n}, \quad \forall t \in \mathbf{R} .
$$

Следовательно, $I(n)=0$, когда $\Delta_{n}=0$. Если $\Delta_{n}>0$, то, полагая $\alpha_{n}=\left(n / \Delta_{n}\right)^{1 / 2}$, мы получим

$$
\begin{aligned}
n I(n) & \leqslant \frac{c n}{h_{n}}\left[\int_{|t| \leqslant \alpha_{n}}\left|p_{n}(t)-q_{n}(t)\right| \frac{1}{t^{2}} d t+\int_{|t|>\alpha_{n}} \frac{2}{t^{2}} d t\right] \\
& \leqslant \frac{c}{h_{n}}\left[\Delta_{n} \alpha_{n}+\frac{n}{\alpha_{n}}\right]=\operatorname{ch}_{n}^{-1}\left(n \Delta_{n}\right)^{1 / 2}
\end{aligned}
$$


в силу (5.20)-(5.22). Следовательно, отсюда и из леммы 8 вытекает (5.17).

Доказательство теоремы 1 завершено.

3 а м е ч а н и е 3 . Условие $\theta>5$ в предположении $2^{\circ}$ может быть ослаблено до условия $\theta \geqslant 5$ ценой удлинения доказательства. Укажем основной шаг доказательства. Воспользовавшись тем же самым приемом урезания, что и в п. 4 (см. абзац между (4.5) и (4.6)), мы можем доказать, что лемма 7 справедлива при замене $m_{n}=O\left(n^{1-\delta}\right)$ на $m_{n}=O(n / \log n)$.

\section{СПИСОК ЛИТЕРАТУРЫ}

1. Alam K., Saxena K.M.L. Positive dependence in multivariate distributions. Comm. Statist. Theory Methods, 1981, v. A10, p. 1183-1196.

2. Hu T. C., Weber $N$. C. On the rate of convergence in the strong law of large numbers for arrays. - Bull. Austral. Math. Soc., 1992, v. 45, № 3, p. 479-482.

3. Joag-Dev K., Proschan F. Negative association of random variables, with applications. - Ann. Statist., 1983, v. 11, p. 286-295.

4. Li D. L., Rao B., Tomkins J. A strong law for $B$-valued arrays. - Proc. Amer. Math. Soc., 1995 , v. 123 , № 10, p. 3205-3212.

5. Matula $P$. A note on almost sure convergence of sums of negatively dependent random variables. - Statist. Probab. Lett., 1992, v. 15, p. 209-213.

6. Newman C. M. Asymptotic independence, limit theorem for positively and negatively dependent random variables. - Inequalities in Statistics and Probability. Ed. by Y.L. Tong. Hayward: IMS, 1984, p. 127-140. (IMS Lecture Notes Monograph Ser., v. 5.)

7. Pan J.M. On the convergence rate in the central limit theorem for negatively associated sequences. - Chinese J. Appl. Probab. Statist., 1997, v. 13, p. 183-192.

8. Петров В. В. Суммы независимых случайных величин. М.: Наука, 1972, 416 с.

9. $Q i$ Y. C. On the strong convergence of arrays. - Bull. Austral. Math. Soc., 1994, v. 50, № 2, p. 219-223.

10. Qi Y.C. On the one-sided logarithmic law for arrays. - Systems Sci. Math. Sci., 1999 , v. 12, p. 123-132.

11. Shao Q.M. A comparison theorem on moment inequalities between negatively associated and independent random variables. - J. Theoret. Probab., 2000, v. 13, № 2 , p. 343-356.

12. Su C., Chi X. Some results on CLT for nonstationary NA sequences. - Acta Math. Appl. Sinica, 1998, v. 21, p. 10-21 (in Chinese).

13. Su C., Zhao L.C., Wang Y.B. Moment inequalities and weak convergence for negatively associated sequences. - Sci. China Ser. A, 1997, v. 40, p. 172-182.

14. Wittmann R. A general law of iterated logarithm. - Z . Wahrscheinlichkeitstheor. verw. Geb., 1985, B. 68, S. 521-543. 